\title{
Risk Factors of Elderly Type 2 Diabetes Mellitus Complicated with Osteoporosis and Effects of Vitamin K1 on Bone Mineral Density and Insulin Resistance
}

\author{
Lei Sun ${ }^{1, ~ *, ~ H o n g j i a n ~ Y u ~}{ }^{1}$, Donghui Tian ${ }^{2}$, Rui Zhang ${ }^{3}$, Wenjing Du ${ }^{4}$ \\ ${ }^{1}$ Department of Orthopedics, Binzhou People's Hospital, Binzhou City, P.R.China \\ ${ }^{2}$ Department of Neurology, Binzhou People's Hospital, Binzhou City, P.R.China \\ ${ }^{3}$ Technical Office, People's Court of Bincheng District, Binzhou City, P.R.China \\ ${ }^{4}$ Technical Office, Binzhou Intermediate People's Court, Binzhou City, P.R.China
}

Email address:

LeiSun2019@163.com (Lei Sun)

${ }^{*}$ Corresponding author

\section{To cite this article:}

Lei Sun, Hongjian Yu, Donghui Tian, Rui Zhang, Wenjing Du. Risk Factors of Elderly Type 2 Diabetes Mellitus Complicated with Osteoporosis and Effects of Vitamin K1 on Bone Mineral Density and Insulin Resistance. American Journal of Life Sciences. Vol. 7, No. 1, 2019, pp. 12-16. doi: 10.11648/j.ajls.20190701.13

Received: January 22, 2019; Accepted: February 27, 2019; Published: March 8, 2019

\begin{abstract}
Aim: To analyze the bone mineral density (BMD) changes of type 2 diabetes mellitus (T2DM) after being complicated with osteoporosis (OP) and their correlations with multiple risk factors. Methods: 240 cases of elderly T2DM patients were divided into an OP group and a non-OP group according to the BMD values. The results were subjected to correlation analyses. Thereafter the 120 patients in the OP group were randomly divided into three groups to be treated with alfacalcidol (group A), vitamin K1 (group B) and alfacalcidol plus vitamin K1 (group C) continuously for 12 months. The BMD, FBG levels, fasting insulin (FINS) levels, prothrombin time (PT), activated partial thromboplastin time (APTT), thrombin time (TT) and fibrinogen (FIB) levels were measured 0, 6 and 12 months after administration. The heights, weights, BMI and HOMA-insulin resistance (HOMA-IR) of the patients were measured by designated personnel. Results: The patients in the OP group were of older age, longer disease course, lower BMD, and higher serum phosphorus than those in the non-OP group. The results of the two groups differed significantly $(\mathrm{P}<0.05)$. The BMD of T2DM patients was negatively correlated with age, disease course, ALP and HbAlc and positively correlated with BMI. All the treatment methods elevated the BMD values of the three groups after 12 months $(\mathrm{P}<0.05$ or $\mathrm{P}<0.01)$, and those of group $\mathrm{C}$ were elevated more significantly than those of group $\mathrm{A}$ and group $\mathrm{B}(\mathrm{P}<0.05$ or $\mathrm{P}<0.01)$. Although the number of lumbar vertebrae L1-L4 and ectotrochanter of group A patients were higher than that of group $B$ patients after being treated for 3 months $(P<0.01)$, the results of the two groups did not differ significantly ( $\mathrm{P}>0.05) 12$ months after treatment. Conclusions: Old age, low BMI, long disease course, poor blood glucose control and high serum ALP are the risk factors leading to T2DM complicated with OP. However, vitamin K1, which increased the BMD of T2DM patients and boosted insulin resistance, could be combined with alfacalcidol and calcium supplement owing to the lack of abnormal blood clotting mechanism after long-term administration.
\end{abstract}

Keywords: Bone Mineral Density, Type 2 Diabetes Mellitus, Osteoporosis, Insulin Resistance

\section{Introduction}

More people are suffering from diabetes mellitus and osteoporosis (OP) owing to unhealthy life style and aging issues [1]. Diabetic osteoporosis (DOP) is a systemic metabolic disease of bone loss and easy fracture resulting from the altered bone structures owing to diabetes [2]. Type 2 diabetes (T2DM) endangers health and leads to heavy economic burden. The relationships between bone mineral density (BMD) and relative metabolism indexes of the T2DM patients enrolled or treated were observed to prevent DOP. Vitamin $\mathrm{K}$ has been commonly used to treat abnormal blood clotting function and bleeding disorders. Recently, some 
epidemiological studies have revealed that insufficient vitamin $\mathrm{K}$ leads to reduced $\mathrm{BMD}$ and increased risks of fracture. However, vitamin $\mathrm{K}$ has seldom been utilized to treat OP. Therefore, elderly T2DM patients complicated with OP were administered with regular doses of vitamin $\mathrm{K} 1$ to explore the effects on BMD and insulin resistance [3].

\section{Materials and Methods}

\subsection{General Information}

240 T2DM patients enrolled and treated from January 2010 to January 2011 were selected, consisting of 120 cases of T2DM complicated with OP and 120 cases of T2DM. The patients aging 53.76-74.23 years old (average: $63.44 \pm 10.61$ ) comprised 110 males and 130 females with the disease course of 1.33-9.43 years (average: 10.25 \pm 9.14 ). They were diagnosed according to the WHO diabetes mellitus standard in 1999 [4]: diabetes symptoms + plasma glucose level at any time $\geq 11.1 \mathrm{mmol} / \mathrm{L}$, fasting blood glucose $(\mathrm{FBG})$ level $>7.0$ $\mathrm{mol} / \mathrm{L}$, or OGTr $2 \mathrm{~h}$ plasma glucose level $\geq 1.1 \mathrm{mmol} / \mathrm{L}$. Exclusion standards of diabetes mellitus: gestational diabetes mellitus and type 1 diabetic mellitus (T1DM). Exclusion standards of diseasing leading to secondary OP: gonadal dysfunction, Cushing syndrome, hyperthyroidism, hyperparathyroidism, malnutrition, bone marrow fibrosis, chronic kidney failure and leukemia; without diabetes ketoacidosis, high-permeability non-ketotic coma and other acute complications; severe liver and kidney diseases, other bone metabolism diseases and bone metabolism medicine effects; administration of calcium agents, lipid-regulating drugs and active vitamin D in the last 4 weeks.

\subsection{Methods}

\subsubsection{Metabolism Indexes and BMD Grouping}

240 elderly T2DM patients enrolled from January 2010 to January 2011 were were divided into an OP group (130 cases) and a non-OP group (110 cases) according to the BMD values. The genders, ages, body mass indexes (BMI), T2DM disease course, levels of FBG, postprandial blood glucose (PBG), hemoglobin A1c (HbA1c), low-density lipoprotein cholesterol (LDL-C), triglycerides (TG), serum calcium, phosphorus and magnesium, alkaline phosphatase (ALP) and uric acid of the two groups were compared. The results were subjected to correlation analyses.

Determination of BMD

BMD values were measured by Lunar DPX-NT X-ray bone densitometry (GE, USA), the quality of which was controlled by designated personnels. The BMD values of lumbar vertebra L4 were measured and averaged, and the $\mathrm{T}$ values were calculated acoording to the WHO standard in 1994: T value $=$ measured BMD - average BMD of healthy youths. The standard derivation of healthy yough BMD is SD. T value $>$ $-1.0 \mathrm{SD}$ is determined as healthy, $-2.5 \mathrm{SD}<\mathrm{T}$ value $<-1.0 \mathrm{SD}$ is determined as bone loss, $\mathrm{T}$ value $<-2.5 \mathrm{SD}$ is determined as $\mathrm{OP}$, and $\mathrm{T}$ vlue $<-2.5 \mathrm{SD}$ complicated with $\mathrm{OP}$ facture is determined as severe OP.

\subsubsection{Determination of Biochemical Indexes and BMI}

1) Blood glucose: The venous plasma blood glucose levels were determined by designated personnels; 2) HbA1c: HbA1c levels were measured by high pressure liquid chromatography (HPLC). The reagents were purchased from Sigma with the intra-batch and inter-batch variabilities of $<9 \%$ and $<15 \%$; 3 ) Blood lipid: The TG and LDL-C levels in fasting venous blood were measured according to the instructions of kits (Sigma, USA); 4) Serum calcium, phosphorus and magnesium and ALP: Their levels were measured by an automatic biochemical analyzer; 5) Blood uric acid: The levels were determined by the uricase-POD method according to the kit instruction utilizing a Beckman DxC600 automatic biochemical analyzer; 6) BMI: The BMI values were calculated as: $\mathrm{BMI}=$ body mass $(\mathrm{kg}) /$ height $^{2}\left(\mathrm{~m}^{2}\right)$.

\subsection{Effects of Vitamin K1 on BMD and Insulin Resistance}

\subsubsection{Treatment Grouping}

120 patients enrolled from January 2010 to January 2011 suffering from T2DM complicated with OP were randomly divided into 3 groups: Group A: 40 cases (11 males, 29 females), average $(62.3 \pm 10.6)$ years old; group B: 40 cases $(10$ males, 30 females), average $(60.7 \pm 11.3)$ years old; group $C$ : 40 cases (12 males, 28 females), average (57.5 \pm 9.8 ) years old. The ages and genders of the two groups did not differ significantly $(\mathrm{P}>0.05)$. All patients accord with the diabetes and OP diagnosis standards of WHO in 1999. They had been orally administered with antidiabetic agents. All women had entered menopause.

\subsubsection{Treatment Method}

Group A were treated with alfacalcidol $(0.5 \mu \mathrm{g}, \mathrm{qd})$, group B were treated with vitamin $\mathrm{K} 1$ (100 mg, tid), and group C were treated with alfacalcidol plus vitamin K1. All patients were supplemented with $450 \mathrm{mg}$ calcium tablets (qd). The treatments were lasted continuously for 12 months. The BMD, FBG levels, fasting insulin (FINS) levels, prothrombin time (PT), activated partial thromboplastin time (APTT), thrombin time (TT) and fibrinogen (FIB) levels were measured 0, 6 and 12 months after administration. The heights, weights, BMI and HOMA-insulin resistance (HOMA-IR) of the patients were measured by designated personnels. HOMA-IR $=\operatorname{lnFBG} \times \mathrm{FINS} / 22.5$. The BMD values of femoral neck, femoral torch, femoral Ward's triangle and lumbar vertebra L1-L4 were measured. The machine with the accuracy of $1 \%$ and the measurement error of $<1 \%$ was corrected prior to measurement every day.

\subsection{Statistical Analysis}

The data were analyzed by SPSS 18.0. The measurement data were expressed as mean $\pm \mathrm{SD}$. The mean values of the two groups were compared by the t test. $\mathrm{P}<0.05$ was considered statiscically significant.

\section{Results}

\subsection{Metabolism Indexes Comparison}

Table 1 and 2 show that the T2DM patients complicated 
with OP are older with longer disease course, lower BMD and

$(\mathrm{P}<0.05)$ higher serum phosphorus levels compared to those without OP

Table 1. General information, $B M D, F B G, P B G$ and serum calcium of the two groups ( $x \pm s)$.

\begin{tabular}{|c|c|c|c|c|c|c|c|c|}
\hline Group & Gender (M/F) & $\begin{array}{l}\text { Age (years } \\
\text { old) }\end{array}$ & $\begin{array}{l}\text { Disease } \\
\text { course (year) }\end{array}$ & BMI $\left(\mathrm{kg} / \mathrm{m}^{2}\right)$ & $\operatorname{BMD}\left(\mathrm{g} / \mathrm{m}^{2}\right)$ & $\begin{array}{l}\text { FBG } \\
(\mathrm{mmol} / \mathrm{L})\end{array}$ & $\begin{array}{l}\text { PBG } \\
(\mathrm{mmol} / \mathrm{L})\end{array}$ & $\begin{array}{l}\text { Serum calcium } \\
(\mathrm{mmol} / \mathrm{L})\end{array}$ \\
\hline OP & $44 / 86$ & $66.15 \pm 9.46$ & $11.43 \pm 10.56$ & $26.03 \pm 3.80$ & $0.78 \pm 0.07$ & $4.50 \pm 3.45$ & $10.76 \pm 8.03$ & $2.35 \pm 0.11$ \\
\hline Non-OP & $65 / 45$ & $60.51 \pm 10.59$ & $8.88 \pm 7.05$ & $26.40 \pm 4.14$ & $1.22 \pm 0.08$ & $4.17 \pm 3.38$ & $9.01 \pm 8.20$ & $2.35 \pm 0.11$ \\
\hline$P$ value & & 0.000 & 0.007 & 0.531 & 0.000 & 0.040 & 0.056 & 0.559 \\
\hline
\end{tabular}

Table 2. Biochemical indexes of the two groups $(x \pm s)$.

\begin{tabular}{|c|c|c|c|c|c|c|c|}
\hline Group & HbA1c\% & TG (mmol/L) & $\begin{array}{l}\text { LDL-C } \\
(\mathrm{mmol} / \mathrm{L})\end{array}$ & $\operatorname{ALP}(\mathbf{U} / \mathbf{L})$ & $\begin{array}{l}\text { Serum phosphorus } \\
(\mathrm{mmol} / \mathrm{L})\end{array}$ & $\begin{array}{l}\text { Serum magnesium } \\
(\mathrm{mmol} / \mathrm{L})\end{array}$ & $\begin{array}{l}\text { Uric acid } \\
(\mathrm{mmol} / \mathrm{L})\end{array}$ \\
\hline OP & $8.75 \pm 2.24$ & $1.61 \pm 1.05$ & $2.55 \pm 1.15$ & $67.77 \pm 24.17$ & $1.11 \pm 0.16$ & $0.78 \pm 0.69$ & $323.33 \pm 1.2 .65$ \\
\hline Non-OP & $8.50 \pm 2.37$ & $1.76 \pm 1.21$ & $2.43 \pm 1.16$ & $64.68 \pm 24.10$ & $1.07 \pm 0.15$ & $0.78 \pm 0.05$ & $325.03 \pm 83.77$ \\
\hline $\mathrm{t}$ value & -0.997 & 4.138 & 2.664 & -0.625 & -20.755 & -0.840 & -1.907 \\
\hline$P$ value & 0.316 & 0.158 & 0.518 & 0.250 & 0.020 & 0.655 & 0.863 \\
\hline
\end{tabular}

\subsection{Correlation Analysis of DOP Risk Factors}

The BMD values of T2DM patients are negatively correlated with age, disease course, and HbAlc and ALP levels, they are positively correlated with BMI (Table 3), and they are not correlated with the levels of FBG, PBG, TG, LDL-C, serum calcium, phosphorus and magnesium, and uric acid $(\mathrm{r}=-0.017,-0.047,-0.091,-0.048,0.089,0.065,-0.089$, $-0.068, \mathrm{P}>0.05)$.

Table 3. Correlation analysis of DOP risk factors.

\begin{tabular}{|c|c|c|c|c|c|}
\hline Index & Age (years old) & Disease course (year) & BMI $\left(\mathrm{kg} / \mathrm{m}^{2}\right)$ & $\operatorname{ALP}(\mathbf{U} / \mathbf{L})$ & HbA1c (\%) \\
\hline r value & -0.139 & -0.009 & 0.038 & -0.060 & -0.010 \\
\hline$P$ value & 0.007 & 0.000 & 0.018 & 0.019 & 0.013 \\
\hline
\end{tabular}

\subsection{General Information and Biochemical Indexes Before and After the Administration of Vitamin K1}

The BMI, PT, APTT, TT and FIB of the two groups and those in each group after 0,6 and 12 months of treatment all did not differ significantly ( $\mathrm{P}>0.05)$. The HOMA-IR levels of group A and group $\mathrm{B}$ that did not change apparently after 6 months of treatment $(\mathrm{P}>0.05)$ significantly decreased 6 months later $(\mathrm{P}<0.05)$, while those of group $\mathrm{C}$ that had already decreased apparently after 6 months of treatment $(\mathrm{P}<0.01)$ were further lowered 6 months later $(\mathrm{P}<0.05)$. The HOMA-IR levels of group $\mathrm{C}$ were significantly higher than those of group A and group B, but the results of group A and group B did not differ significantly $(\mathrm{P}>0.05)$. The general information and biochemical indexes before and after treatment are shown in Table 4.

Table 4. General information and biochemical indexes before and after treatment ( $x \pm s)$.

\begin{tabular}{|c|c|c|c|c|c|c|c|c|c|}
\hline & \multicolumn{3}{|l|}{ Group A } & \multicolumn{3}{|l|}{ Group B } & \multicolumn{3}{|l|}{ Group C } \\
\hline & $\mathbf{0}$ & 6 & 12 & 0 & 6 & 12 & $\mathbf{0}$ & 6 & 12 \\
\hline $\mathrm{BMI} /\left(\mathrm{kg}^{*} \mathrm{~m}^{-2}\right)$ & $23.0 \pm 4.54$ & $22.8 \pm 5.00$ & $22.1 \pm 4.06$ & $21.8 \pm 4.17$ & $22.5 \pm 4.14$ & $23.6 \pm 5.02$ & $21.7 \pm 4.32$ & $22.4 \pm 4.98$ & $22.9 \pm 4.66$ \\
\hline HOMA-IR & $6.91 \pm 1.10$ & $6.52 \pm 1.05$ & $6.00 \pm 1.08 \boldsymbol{\Delta}$ & $7.00 \pm 1.01$ & $6.74 \pm 1.04 \Delta$ & $5.87 \pm 1.08 \boldsymbol{\Delta} \square$ & $7.02 \pm 1.06$ & $5.70 \pm 1.05 \Delta \diamond \#$ & $5.11 \pm 1.07 \boldsymbol{\Delta} \square *$ \\
\hline $\mathrm{PT} / \mathrm{s}$ & $10.05 \pm 1.07$ & $9.90 \pm 1.11$ & $10.10 \pm 1.19$ & $10.08 \pm 1.17$ & $10.19 \pm 1.06$ & $10.06 \pm 1.16$ & $10.12 \pm 1.14$ & $10.07 \pm 1.18$ & $10.10 \pm 1.15$ \\
\hline $\mathrm{APTT} / \mathrm{s}$ & $26.72 \pm 4.11$ & $28.01 \pm 5.00$ & $27.11 \pm 5.04$ & $28.20 \pm 5.15$ & $26.71 \pm 5.01$ & $27.38 \pm 5.06$ & $26.79 \pm 5.12$ & $28.03 \pm 5.18$ & $27.79 \pm 5.15$ \\
\hline $\mathrm{TT} / \mathrm{s}$ & $17.15 \pm 2.05$ & $18.33 \pm 2.10$ & $17.55 \pm 2.28$ & $18.00 \pm 2.13$ & $18.18 \pm 2.11$ & $18.05 \pm 2.05$ & $17.99 \pm 2.35$ & $18.01 \pm 2.07$ & $17.88 \pm 2.15$ \\
\hline $\mathrm{Fib} / \mathrm{G}^{*} \mathrm{~L}$ & $3.11 \pm 0.55$ & $3.21 \pm 0.75$ & $3.00 \pm 0.76$ & $3.18 \pm 0.65$ & $3.05 \pm 0.55$ & $3.10 \pm 0.66$ & $3.08 \pm 0.55$ & $2.99 \pm 0.69$ & $3.12 \pm 0.63$ \\
\hline
\end{tabular}

Comparison between the results after treatment and 0-month results: $\Delta \mathrm{P}<0.05, \boldsymbol{\Delta} \mathrm{P}<0.01$; comparison between 12-month results and 6-month results: $\square \mathrm{P}<0.05$, $\square \mathrm{P}<0.01$; compared to group $\mathrm{A}$ at the same time: $\diamond \mathrm{P}<0.05, \diamond \mathrm{P}<0.01$; compared to group $\mathrm{B}$ at the same time: $* \mathrm{P}<0.05, \# \mathrm{P}<0.01$.

\subsection{BMD Before and After the Administration of Vitamin K1}

The BMD values of all 3 groups were elevated after 6 months of treatment $(\mathrm{P}<0.05)$, and those of group $\mathrm{C}$ were elevated more significantly $(\mathrm{P}<0.05)$. The $\mathrm{BMD}$ values of
L1-L4 and torch of group B were higher than those of group A after 3 months of treatment $(\mathrm{P}<0.01)$, but the values of the two groups did not differ significantly any longer 3 months later $(\mathrm{P}>0.05)$. The BMD values before and after treatment are summarized in Table 5. 
Table 5. BMD values before and after treatment.

\begin{tabular}{|c|c|c|c|c|c|c|c|c|c|}
\hline & Group A & & & Group B & & & Group C & & \\
\hline & 0 & 6 & 12 & 0 & 6 & 12 & 0 & 6 & 12 \\
\hline $\mathrm{L}_{1}-\mathrm{L}_{4}$ & $0.85 \pm 0.04$ & $0.89 \pm 0.04 \Delta$ & $0.94 \pm 0.06 \boldsymbol{\Delta} \square$ & $0.82 \pm 0.07$ & $0.85 \pm 0.05 \Delta$ & $0.94 \pm 0.05 \Delta \mathbf{\Delta}$ & $0.85 \pm 0.06$ & $0.92 \pm 0.04 \mathbf{\Delta} \diamond$ & $0.98 \pm 0.06 \Delta \mathbf{\Delta} \#$ \\
\hline Neck & $0.73 \pm 0.06$ & $0.78 \pm 0.05 \boldsymbol{\Delta}$ & $0.87 \pm 0.05 \boldsymbol{\Delta} \mathbf{\square}$ & $0.72 \pm 0.04$ & $0.79 \pm 0.04 \boldsymbol{\Delta}$ & $0.88 \pm 0.05 \Delta \mathbf{\square}$ & $0.72 \pm 0.05$ & $0.88 \pm 0.03 \boldsymbol{\Delta}$ & $0.93 \pm 0.04 \boldsymbol{\Delta} \mathbf{\nabla} \#$ \\
\hline Torch & $0.53 \pm 0.07$ & $0.57 \pm 0.05 \Delta$ & $0.63 \pm 0.08 \Delta \square$ & $0.58 \pm 0.06$ & $0.64 \pm 0.06 \boldsymbol{\Delta}$ & $0.68 \pm 0.08 \boldsymbol{\Delta} \square$ & $0.55 \pm 0.06$ & $0.65 \pm 0.06 \boldsymbol{\Delta}$ & $0.72 \pm 0.05 \Delta \mathbf{\Delta} \#$ \\
\hline Ward & $0.48 \pm 0.06$ & $0.52 \pm 0.06 \Delta$ & $0.56 \pm 0.07 \boldsymbol{\Delta} \square$ & $0.49 \pm 0.07$ & $0.51 \pm 0.06 \Delta$ & $0.57 \pm 0.08 \Delta \square$ & $0.50 \pm 0.05$ & $0.56 \pm 0.06 \Delta \diamond$ & $0.63 \pm 0.06 \boldsymbol{\Delta} \mathbf{\nabla} \#$ \\
\hline
\end{tabular}

Comparison between the results after treatment and 0-month results: $\Delta \mathrm{P}<0.05, \mathbf{\Delta} \mathrm{P}<0.01$; comparison between 12 -month results and 6-month results: $\square \mathrm{P}<0.05$, $\square \mathrm{P}<0.01$; compared to group $\mathrm{A}$ at the same time: $\diamond \mathrm{P}<0.05, \diamond \mathrm{P}<0.01$; compared to group $\mathrm{B}$ at the same time: $* \mathrm{P}<0.05, \# \mathrm{P}<0.01$.

\section{Discussion}

OP is a senior disease resulting from complicated reasons, which is manifested as reduced bone mechanical strength, bone resorption and loss of coupling during bone formation [5]. It has been reported that the BMD values of senior male T2DM are drastically dropping. Their bone metabolisms are featured in increased bone resorption and inhibited bone formation. The BMD values gradually decrease with prolonged T2DM course [6]. Besides, diabetes patients complicated with nephropathy are prone to fracture. DOP, which is a secondary OP, raises the risk of fracture and threatens the quality of life [7]. OP is classified into primary and secondary ones, and the primary OP is also classified into type I and type II ones. Type I primary OP refers to menopause OP, and type II one refers to that found in the elders over 60 years old. Twice as many women as men are subjected to primary OP. Secondary OP is associated with endocrine disorders, alcohol poisoning, medicine administration, malnutrition, and liver and kidney diseases, etc. [8].

Disease course, which is one of the risk factors leading to DOP, lowers BMD by $10 \%$ every 15 years. Initially, T2DM is manifested as insulin resistance [9], which then results in hyperglycemia [10]. Insulin alters the skeleton structures of diabetes patients by many pathways [11]. The facilitated synthesis of osteocalcin and the accumulation of amino acids in bone cells cooperate with $\mathrm{PTH}$ in regulating the activity of 1-hydroxylase, which promotes the syntheses of 1,25-(OH)D and adenyl cyclase, thereby leading to the deposition of calcium salts. Insufficient insulin excretion also results in OP by reducing the bone transformation rate and inhibiting the synthesis of osteocalcin. In this study, the BMD values of DOP patients were negatively correlated with their disease course $(r=-0.010, P=0.000)$ [12]. The sharp increase of blood glucose cannot increase the synthesis of osteoprotegerin, the levels of which can be lowered by hyperinsulinemia.

BMI is an index reflecting the obesity degree. Although obesity protects bones during menopause, it also affects the peak bone mass. Obesity also helps men in stimulating the excretion of insulin-like growth factors (IGF-1), which activates the bone cells [13]. IGF-1 also avoids the formation of sex hormone-binding globulin, which increases the free sexual hormones, thereby inhibiting bone resorption and preventing OP. In addition, obesity is conducive to the T2DM patients over 65 years old. The blood glucose levels are monitored by $\mathrm{HbA} 1 \mathrm{c}$ which reflects the average blood glucose levels in recent 23 months. HbAlc that is negatively correlated with BMD is another risk of DOP. Meanwhile, hyperglycemia is relevant to bone transformation [14], which may eventually result in continuous DOP that increases the saccharificated final products and decreases the adhesion of bone cells to bone collagen. The activity of osteoclasts can be increased by varying the saccharificated final products of osteoblasts. OP is triggered by apoptotic osteoblasts via cytoplast pathway and mitogen-activated protein kinase pathway. Moreover, hyperglycemia is able to inhibit the reproduction, maturity and differentiation of osteoblasts both in vitro and in vivo, and to accelerate the apoptosis of bone lining cells. It has also been reported that diabetes, especially in the first two years, is negatively correlated with BMD, suggesting that early control of blood glucose levels can postpone DOP. OP leads to severe pain, fracture, and even deformity, which greatly impact the quality of life and burden diabetes patients economically.

On the other hand, vitamin $\mathrm{K} 1$ is able to regulate bone metabolism, elevate BMD, and prevent bone fracture [15]. In this study, the BMD values of all patients were significantly elevated after 12 months of treatment. The BMD values of L1-L4 and torch of group B were higher than those of group A after 3 months of treatment, but the values of the two groups did not differ significantly any longer 9 months later. The results herein indicate that vitamin K1 could treat DOP, its effect on BMD enhancement was similar to alfacalcidol though. The combination of vitamin $\mathrm{K} 1$ and alfacalcidol functioned significantly better than the individual administrations, revealing that vitamin $\mathrm{K} 1$ and alfacalcidol functioned synergetically. Vitamin K1 elevates BMD probably by participating in the r-carboxylation of glutamic acid in osteocalcin that facilitates bone mineral salts deposition and bone formation, reducing the excretion of uric calcium that influences bone metabolism, and inhibiting the activation factors of bone resorption, such as interleukin (IL)-1 and IL-6 [16]. In this study, the HOMA-IR levels of all 3 groups were significantly lowered after 12 months of treatment. The HOMA-IR levels of group C were significantly higher than those of group A and group B, but the results of group A and group B did not differ significantly. Considering that vitamin $\mathrm{K}$ has long been utilized to treat blood clotting disorders [17], the missing of blood clotting in the patients after being treated for 12 months indicates the security in healthy people [18]. The maintained blood clotting function in the cases of long-term administration of vitamin K1 may be associated with the saturation mechanism, i.e. vitamin $\mathrm{K}$ that is accumulated in liver allows the normal carboxylation of 
hepatogenic coagulation factors, which is thereafter distributed in extrahepatic skeletons that prevents OP [19].

\section{Conclusion}

In summary, vitamin $\mathrm{K} 1$, which increased the $\mathrm{BMD}$ of T2DM patients and boosted insulin resistance, could be combined with alfacalcidol and calcium supplement owing to the lack of abnormal blood clotting mechanism after long-term administration. However, the appropriate doses and security of vitamin $\mathrm{K} 1$ are still in need of further investigation due to the small case number and short observation time.

\section{References}

[1] Takeuchi Y. Diabetes mellitus and osteoporosis. Therapeutic strategy for osteoporosis in patients with diabetes mellitus. Clin Calcium, 2016, 22 (9): 1410-1415.

[2] Williams EA. Folate, colorectal cancer and the involvement of DNA methylation. Proc Nutr Soc, 2017, 71 (4): 592-7.

[3] Melton LJ, Leibson CL, Achenbach SJ, et al. Fracture risk in type 2 diabetes: update of a population-based study. J Bone Miner Res, 2018, 23 (8): 1334.

[4] Watanabe R, Okazaki R. Diabetes mellitus and osteoporosis. Diabetes mellitus and bone and calcium metabolism. Clin Calcium, 2012, 22 (9): 1307-1014.

[5] Xicola RM. Llor X. et al. DNA methylation defects in sporadic and hereditary colorectal cancer [J]. Gastroenterol Hepatol, 2017, 35 (7): 480-7.

[6] Al-Lawati JA, N Barakat M, Al-Zakwani I, et al. Control of risk factors for cardiovascular disease among adults with previously diagnosed type 2 diabetes mellitus: a descriptive study from a middle eastern arab population. Open Cardiovasc Med J, 2018; 6: $133-40$.

[7] Abdulameer SA, Sulaiman SA, Hassali MA, et al. Osteoporosis and type 2 diabetes mellitus: what do we know, and what we can do? Patient Prefer Adherence, 2017; 6: 435-48.

[8] Koroglu BK, Kiris F, Ersoy IH, et al. Relation of leptin, adiponectin and insulin resistance to bone mineral density in type 2 diabetic postmenopausal women. Endokrynol Pol, 2017; 62 (5): 429-35.
[9] Hayakawa N, Suzuki A. Diabetes mellitus and osteoporosis. Effect of antidiabetic medicine on osteoporotic fracture. Clin Calcium, 2018, 22 (9): 1383-1390.

[10] Uenishi K. Diabetes mellitus and osteoporosis. Dietary therapy of diabetes related osteoporosis. Clin Calcium, 2012, 22 (9): 1398-1402.

[11] Yamada S, Inaba M. Diabetes mellitus and osteoporosis. Bone metabolic disorder in diabetic nephropathy. Clin Calcium, 2016, 22 (9): 1333-1341.

[12] Knudsen ST, Jeppesen P, Poulsen PL, et al. Plasma concentrations of osteoprotegerin during normo- and hyperglycaemic clamping. Scand J Clin Lab Invest, 2017, 67: 135-142.

[13] Wongdee K, Charoenphandhu N. Osteoporosis in diabetes mellitus: Possible cellular and molecular mechanisms. World J Diabetes, 2018, 2 (3): 41-48.

[14] Vieru A, Niţă O, Graur LI, et al. Neuropad test in evaluation of diabetic foot. Rev Med Chir Soc Med Nat Iasi, 2018, 116 (1): 90-96.

[15] Yoshida M, Jacques PF, Meigs JB, et al. Effect of vita-min K supplementation on insulin resistance in older men and women. Diabetes Care, 2018, 31 (11): 2092.

[16] Yasuda S, Wada S. Diabetic osteopahty and vitamin K. Clin Calcium, 2016, 16 (8): 1351-1357. Iwamoto J, Yeh JK, Sato Y, et al. Comparative effects of vitamin $\mathrm{K}$ and vitamin $\mathrm{D}$ supplementation on calcium balance in young rats fed normal or lows calcium diets. Nutr Sci Vitaminol (Tokyo), 2017, 51 (4): 211.

[17] Wada S. Therapeutic approaches for diabetic osteopathy. Clin Calcium, 2016, 16 (8): 1297-1304. Roe DA. Drug and nutrient interactions in the elderly diabetic. Drug Nutr Interact, 2018, 5 (4): 195-203.

[18] Kaneda A. Yagi K. et al. Two groups of DNA methylation markers to classify colorectal cancer into three epigenotypes. Cancer Sci, 2017, 102 (1): 18-24.

[19] Kim JW. Park HM. et al. Polymorphisms in genes involved in folate metabolism and plasma DNA methylation in colorectal cancer patients. Oncol Rep, 2018, 25 (1): 167-72. 